\title{
Immune responsive gene 1 , a novel oncogene, increases the growth and tumorigenicity of glioma
}

\author{
JUN PAN $^{1 *}$, XIAOYONG ZHAO ${ }^{2 *}$, CHUNNAN LIN $^{1}$, HONGCHAO XU $^{1}$, \\ ZHILIN YIN $^{1}$, TIANZHU LIU ${ }^{1}$ and SHIZHONG ZHANG ${ }^{1}$ \\ ${ }^{1}$ The National Key Clinic Specialty, The Neurosurgery Institute of Guangdong Province, \\ Guangdong Provincial Key Laboratory on Brain Function Repair and Regeneration, Department of Neurosurgery, \\ Zhujiang Hospital, Southern Medical University; ${ }^{2}$ Department of Neurosurgery, Huadu Hospital, \\ Southern Medical University, Guangzhou, Guangdong 510000, P.R. China
}

Received May 20, 2014; Accepted August 4, 2014

DOI: 10.3892/or.2014.3474

\begin{abstract}
Immune responsive gene 1 (IRG1) is highly expressed in mammalian macrophages during inflammation. However, the role of IRG1 in tumorigenesis remains unclear. In the present study, we aimed to clarify the epigenetic regulation and biological functions of IRG1 in glioma. We found that the expression level of IRG1 influenced the WHO stage in 140 glioma patients. Overexpression of IRG1 increased the growth, invasion, and tumorigenesis in U251 and SHG-44 glioma cells both in vitro and in vivo. Suppression of IRG1 expression by si-IRG1 decreased the levels of cell cycle regulatory proteins, namely, E2F1, p21, CDK4, CDK6 and cyclin D1. Knockdown of IRG1 expression by RNA interference increased E-cadherin expression and decreased the amounts of snail and vimentin. Furthermore, the suppression of IRG1 expression inhibited the expression of $\mathrm{NF}-\kappa \mathrm{B}$ and STAT3, suggesting a role of IRG1 in regulating the genes associated with these factors and thereby contributing to a decrease in glioma cell proliferation, migration and invasion. Collectively, our findings revealed that IRG1 is a candidate oncogene that is amplified in glioma and is involved in novel mechanisms that influence glioma pathogenesis.
\end{abstract}

\section{Introduction}

Glioma is the most common malignant neoplasm worldwide. Even with an increase in the risk and incidence of glioma, it is estimated that $\sim 60 \%$ of subjects over the age of 50 in the USA are not screened regularly (1). In addition, a higher frequency of astrocytoma among African-Americans (40-49

Correspondence to: Professor Shizhong Zhang, Department of Neurosurgery, Zhujiang Hospital, Southern Medical University, Guangzhou, Guangdong 510000, P.R. China

E-mail: shizhongzhang1@gmail.com

*Contributed equally

Key words: glioma, immune responsive gene 1, tumorigenicity, oncogene years of age) as compared to Hispanic-Americans has been reported suggesting an increased susceptibility to glioma risk $(1,2)$. Current treatment is based on surgical excision after combined chemotherapy and radiation therapy, yet the prognosis for glioma patients has not fundamentally improved. The development of glioma commonly consists of simultaneous dysregulation of cell growth, differentiation and migration functions; cell malignant transformation; and metastasis. However, the molecular mechanisms underlying the development of glioma are not completely understood.

Immune responsive gene 1 (IRG1) was originally identified as a 2.3-kb cDNA from a library synthesized from the mRNA isolated from a macrophage cell line after LPS stimulation. Based on sequence homology, IRG1 has been classified as a member of the MmgE/PrpD family (3). IRG1 was shown to play important roles in embryonic implantation and neurodegeneration (4), and to mediate the production of itaconic acid, which contributes to the activity of macrophages during inflammation (5). Recent studies have found that chronic inflammation is implicated in tumorigenesis and cancer progression and is considered as one of the independent risk factors. IRG1 knockdown by siRNA was found to result in significantly increased production of pro-inflammatory cytokines in LPS-tolerized macrophages and the activation of transcription factors $\mathrm{NF}-\kappa \mathrm{B}$ and interferon regulatory factor 3 (IRF3), which was accompanied by the increased generation of reactive oxygen species (ROS) and downregulation of zinc finger protein A20 (6,7). IRG1 expression and subcellular localization in murine macrophages demonstrated a pattern typical of a TNF- and IFN- $\gamma$-co-regulated gene. In murine ANA-1 macrophages, IRG1 expression was shown to be induced by several pro-inflammatory cytokines and Toll-like receptor (TLR) agonists (8). TLRs are upregulated in many tumor cell lines and tissues, especially in the tumors of epithelial origin, such as ovarian and breast cancers (9). Recent studies show that the expression of TLRs on malignant and immune cells can promote inflammation and cell survival in the tumor microenvironment. A variety of endogenous TLR ligands can activate TLR signaling pathways, inducing nuclear export of tumorigenic transcription factors and secretion of pro-inflammatory cytokines such as interleukin-6 
(IL-6), chemokine ligand 2 (CCL-2), and vascular endothelial growth factor (VEGF), thereby promoting tumor development, angiogenesis, invasion, metastasis and evasion of immune surveillance $(10,11)$.

In the present study, we investigated the role of IRG1 in the pathogenesis of glioma and examined the association of IRG1 expression with glioma clinicopathologic characteristics, including survival of cancer patients. We identified IRG1-induced effects on cell growth, invasion, and in vivo tumorigenesis, confirmed increased IRG1 expression in primary glioma tissues and cell lines, and found a direct correlation between IRG1 levels and the clinical outcome of glioma patients.

\section{Materials and methods}

Cell lines and siRNA transfection. Human glioma cell lines (A172, U251, U87, SHG-44, TJ-105, H4 and U118MG) were obtained from the the Neurosurgery Institute of Guangdong Province (Guangzhou, China). The U-373MG cell line was purchased from the American Type Culture Collection. Both the U251 and U87 cell lines were kind gifts from Professor Xin-Yuan Guan (Sun Yat-Sen University Cancer Center, Guangzhou, China). All cell lines were cultured in Dulbecco's modified Eagle's medium with $10 \%$ fetal bovine serum (FBS) and incubated in a humidified atmosphere of $5 \% \mathrm{CO}_{2}$ and $95 \%$ air at $37^{\circ} \mathrm{C}$. Transfection of siRNA was performed by RNAi-Max (Invitrogen Life Technologies, Carlsbad, CA, USA) according to the manufacturer's protocol. The siRNA sequences are provided upon request. In brief, transfection with the final concentration of $50 \mathrm{nM}$ siRNA was conducted when the cell density was $\sim 40 \%$ in the 6 -well plates.

In vivo animal models. This study was carried out in strict accordance with the recommendations in the Guide for the Care and Use of Laboratory Animals of the National Institutes of Health. The protocol was approved by the Committee on the Ethics of Animal Experiments of the Southern Medical University. All surgery was performed under sodium pentobarbital anesthesia, and all efforts were made to minimize suffering.

Acquisition of clinical specimens. Clinical specimens were obtained from archived tissue samples derived from patients with glioma who underwent surgical treatment at The First Affiliated Hospital of Zhengzhou University, China from January, 2003 through December, 2013. Glioma was diagnosed according to the 2007 WHO Classification of Tumors of Glioblastoma. All tumors were staged on the basis of the pathologic Tumor-Node-Metastasis classification of the International Union Against Cancer $(10,11)$. The selection criteria were as follows: i) the subject presented with a diagnosis of glioma and no history of other tumors; ii) the subject had complete demographic and clinical data, such as age, gender, clinical manifestations, tumor size, extent of resection, adjuvant therapy and date of relapse and/or death; iii) the subject underwent evaluation by enhanced head MRI scanning for tumor relapse or progression after surgery at least once every six months. Written informed consent of the patients was provided by their legal surrogates to permit surgical proce- dures and use of resected tissues. This study was approved by the Specialty Committee on Ethics of Biomedicine Research, Zhengzhou University of China. Human tissue acquisition and use in this study complied with the National Regulations on the Use of Clinical Samples in China.

Collection of clinical information and follow-up. Data were collected by review of the clinical history. Information was recorded including the patient characteristics, relevant symptoms or history, tumor characteristics, extent of resection, post-surgical treatment protocol, overall survival time and progression-free survival time. The follow-up was conducted by telephone or direct correspondence. Postsurgical treatment, including adjuvant radiotherapy and chemotherapy, was fully discussed with the patient or their relatives. The time of tumor relapse or death was verified by the patient or their relatives, by medical recording, or by the social security record. Overall survival (OS) was calculated in months from the date of diagnosis to the time of death, regardless of cause. Progression-free survival (PFS) was defined as the period from the initial date of diagnosis to the time of tumor progression by MRI, or to the time of death of the patient from glioma.

Immunohistochemistry. Paraffin sections $(4 \mu \mathrm{m})$ from samples were deparaffinized in $100 \%$ xylene and re-hydrated in descending ethanol series and water according to standard protocols. Heat-induced antigen retrieval was performed in $10 \mathrm{mM}$ citrate buffer for $2 \mathrm{~min}$ at $100^{\circ} \mathrm{C}$. Endogenous peroxidase activity and non-specific antigen were blocked with peroxidase blocking reagent containing 3\% hydrogen peroxide and serum, followed by incubation with goat anti-human IRG1 antibody (1:100) (Santa Cruz Biotechnology, Santa Cruz, CA, USA) overnight at $4^{\circ} \mathrm{C}$. After washing, the sections were incubated with biotin-labeled rabbit anti-goat antibody for $10 \mathrm{~min}$ at room temperature, and subsequently were incubated with streptavidin-conjugated horseradish peroxidase (HRP) (Maixin Inc., China). The peroxidase reaction was developed using 3,3-diaminobenzidine chromogen solution in DAB buffer substrate. Sections were visualized with DAB and counterstained with hematoxylin, mounted in neutral gum, and analyzed using a bright field microscope.

Western blot analysis. Cells were lysed in RIPA buffer $(50 \mathrm{mM}$ Tris-HCl pH 8.0, 1 mM EDTA pH 8.0, 5 mM DTT, 2\% SDS), and the protein concentration was determined using the BCA assay (Beyotime Inc., China). Total protein $(30 \mu \mathrm{g})$ was resolved using a $10 \%$ SDS-PAGE gel and electro-transferred to polyvinylidene fluoride membranes (Invitrogen), and blocked with 5\% nonfat dry milk in Tris-buffered saline, $\mathrm{pH}$ 7.5. The membranes were immunoblotted overnight at $4^{\circ} \mathrm{C}$ with the following antibodies: rabbit polyclonal anti-IRG1 (1:500), antiE2F1 (1:500), snail (1:1,000), CDK6 (1:1,000), CDK4 (1:1,000), vimentin (1:1,000), E-cadherin and N-cadherin (Santa Cruz Biotechnology). An HRP-conjugated anti-rabbit IgG antibody was used as the secondary antibody (Zhongshan Inc., China).

Colony formation assay. Approximately 100 cells were added to each well of a 6-well culture plate, and each cell group contained 2 wells. After 2 weeks of incubation, cells were washed twice with PBS and stained with Giemsa solution. 


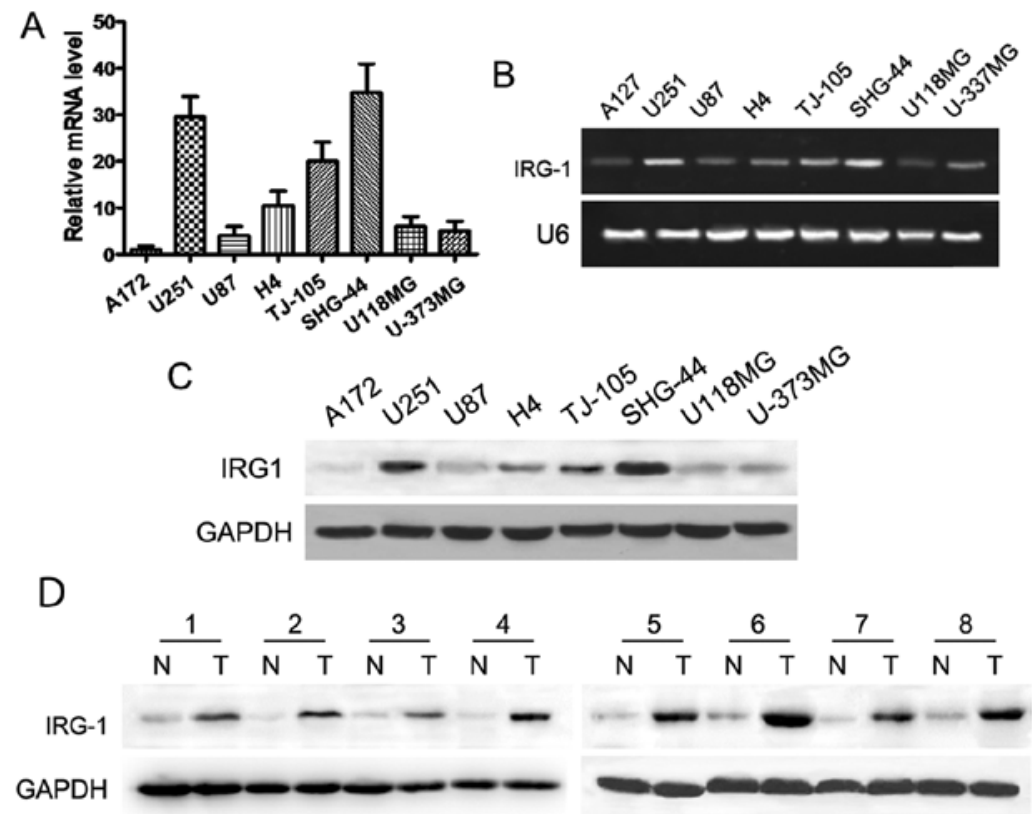

Figure 1. Immune responsive gene 1 (IRG1) is upregulated in human glioma cell lines and clinical specimens. (A and B) Quantitative RT-PCR analysis of IRG1 mRNA expression in 7 human gliomas cell lines. IRG1 mRNA levels were normalized to GAPDH expression. (C) IRG1 protein expression in human glioma cell lines. GAPDH was used as a loading control. (D) IRG1 protein expression in 8 randomly selected paired glioma samples as analyzed by western blotting. T, tumor tissue; $\mathrm{N}$, normal tissue.

The number of colonies containing $\geq 50$ cells was counted under a microscope. The colony formation efficiency was calculated as: Efficiency $=$ (number of colonies/number of cells inoculated) $\mathrm{x} 100 \%$. Each experiment was performed in triplicates.

Cell cycle distribution. To evaluate cell cycle distribution, cells were seeded on $10 \mathrm{~cm}$-diameter plates in RPMI-1640 culture medium containing $10 \%$ NBCS. After $48 \mathrm{~h}$ of incubation, a total of $1 \times 10^{6}$ cells were harvested, rinsed with cold PBS, and fixed with $70 \%$ ice-cold ethanol for $48 \mathrm{~h}$ at $4^{\circ} \mathrm{C}$. Fixed cells were rinsed with cold PBS followed by incubation with PBS containing $10 \mu \mathrm{g} / \mathrm{ml}$ propidium iodide and $0.5 \mathrm{mg} / \mathrm{ml}$ RNase A for $15 \mathrm{~min}$ at $37^{\circ} \mathrm{C}$. The DNA content of the labeled cells was acquired using a FACSCaliber cytometer (BD Biosciences). Each experiment was performed in triplicates.

In vitro cell migration assay. Cells growing in the log phase were treated with trypsin and re-suspended as single-cell solution. A total of $1 \times 10^{5}$ cells were seeded on a fibronectin-coated polycarbonate membrane insert in a Transwell apparatus (Corning Inc., Corning, NY, USA). In the lower chamber, $600 \mu \mathrm{l}$ of RPMI-1640 with $10 \%$ NBCS was added as a chemoattractant. After the cells were incubated for $12 \mathrm{~h}$, the insert was washed with PBS, and cells on the top surface of the insert were removed by a cotton swab. Cells adhering to the lower surface were fixed with methanol, stained with Giemsa, and counted under a microscope in five predetermined fields (x200). All assays were independently repeated at least three times.

Establishment of U251 and SHG-44 cells with stable expression of IRGI short hairpin RNA. The preparation of the lentivirus expressing human IRG1 short hairpin RNA was performed using the pLVTHM-GFP lentiviral RNAi expression system (12). U251 and SHG-44 cells were infected with lentiviral particles containing specific or negative control vectors, and the polyclonal cells with GFP signals were selected for further experiments using FACS flow cytometry.

Statistical analysis. All data were analyzed for statistical significance using SPSS 13.0 software. The Mann-Whitney $\mathrm{U}$ test was applied to the examination of relationship between IRG1 expression levels and clinicopathological characteristics. Survival analysis was performed using Kaplan-Meier method. Multivariate Cox proportional hazards method was used for analyzing the relationship between the variables and patient survival time. One-way ANOVA was used to determine the differences between groups for all in vitro analyses. A P-value of $<0.05$ was considered to indicate a statistically significant result.

\section{Results}

IRG1 is upregulated in human glioma cell lines and clinical specimens. To investigate whether IRG1 plays an important role in gliomas, we examined IRG1 expression in several glioma cell lines A172, U251, U87, SHG-44, TJ-105, H4, U118MG and U-373MG by measuring mRNA and protein levels using realtime RT-PCR and western blotting. Respectively, as shown in Fig. 1A-C the mRNA and protein levels of IRG1 were evidently higher in the U251, SHG-44, TJ-105 and H4 glioma cell lines than the levels in the A251, U118MG and U-373MG cell lines. More importantly, using fresh glioma specimens, we demonstrated that the IRG1 protein level was upregulated in all 8 glioma samples compared to the matched adjacent nontumor tissues (Fig. 1D). 
Table I. Correlation between the clinicopathological characteristics and IRG1 expression in 140 glioma patients.

\begin{tabular}{|c|c|c|c|c|}
\hline \multirow[b]{2}{*}{ Factor } & \multirow[b]{2}{*}{ No. } & \multicolumn{2}{|c|}{ IRG1 protein } & \multirow[b]{2}{*}{ P-value } \\
\hline & & Low, n (\%) & High, n (\%) & \\
\hline \multicolumn{5}{|c|}{ Age (years) } \\
\hline$<60$ & 83 & $47(56.6)$ & $36(43.4)$ & \\
\hline$\geq 60$ & 57 & $24(42.1)$ & $33(57.9)$ & 0.091 \\
\hline \multicolumn{5}{|l|}{ Gender } \\
\hline Male & 68 & $35(51.5)$ & $33(48.5)$ & \\
\hline Female & 72 & $36(50.0)$ & $36(50.0)$ & 0.862 \\
\hline \multicolumn{5}{|c|}{ Tumor size $(\mathrm{cm})$} \\
\hline$<5$ & 49 & $28(57.1)$ & $21(42.9)$ & \\
\hline$\geq 5$ & 91 & $43(47.3)$ & $48(52.7)$ & 0.264 \\
\hline \multicolumn{5}{|c|}{ WHO grade } \\
\hline I & 16 & $5(31.2)$ & $11(68.8)$ & \\
\hline II & 50 & $20(40.0)$ & $30(60.0)$ & \\
\hline III & 34 & $28(70.0)$ & $12(30.0)$ & \\
\hline IV & 40 & $18(45.0)$ & $22(55.0)$ & $0.012^{\mathrm{a}}$ \\
\hline
\end{tabular}

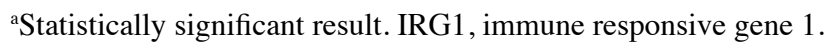

Cytoplasmic IRG1 protein is a prognostic factor for postsurgical glioma patients. The expression levels and subcellular localization of IRG1 protein were examined in 78 archived paraffin-embedded glioma samples and 16 normal epithelium tissues by immunohistochemical staining. The relationship between the clinicopathologic characteristics and IRG1 expression in the glioma patients who underwent surgical resection is presented in Table I. IRG1 was highly expressed in $75.6 \%$ (59/78) of the glioma samples compared to only $37.5 \%(6 / 16)$ of the normal epithelium samples, demonstrating a statistically significant difference $(\mathrm{P}=0.006)$ (Table II). We also observed that IRG1 expression was positively correlated with the WHO grade I, II, III and IV ( $\mathrm{P}=0.012)$ (Table I). Previous results suggest that IRG1 is highly expressed in mammalian macrophages during inflammation, and IRG1 links cellular metabolism with immune defense by catalyzing itaconic acid production (10). Yet, there is no published information as to the expression of IRG1 in relation to cancer prognosis. We, therefore, first stained for IRG1 in glioma tissues in 140 patients. In addition, high (defined as greater than median) IRG1 expression in glioma cells was significantly associated with shorter overall survival $(\mathrm{OS})$ in the entire cohort $(\mathrm{P}<0.05)$.

The staining signal of IRG1 was observed mainly in the different stages of glioma tissues (Fig. 2C-F), and no signals or only weak signals were detected in the adjacent normal brain tissues (Fig. 2A and B). The subcellular location of IRG1 was observed mainly in the cytoplasm of the cancer cells.

The prognostic effect of IRG1 on glioma patient OS was compared between patients with high and low IRG1 protein levels. By Kaplan-Meier curve assessment, patients with a high IRG1 protein level had a significantly lower 5-year survival rate than those with a low IRG1 protein level ( $\mathrm{P}=0.0076$; Fig. 2G).

Furthermore, we compared the relationship between IRG1 expression and recurrence-free survival (RFS). The patients
Table II. Upregulation of IRG1 protein expressin in glioma compared to epithelium tissues.

\begin{tabular}{lcccc}
\hline & & \multicolumn{2}{c}{ IRG1 protein expression } & \\
\cline { 3 - 4 } Group & Total & Low, n (\%) & High, n (\%) & P-value \\
\hline Normal epithelium & 16 & $10(62.5)$ & $6(37.5)$ & 0.006 \\
Glioma & 78 & $19(24.4)$ & $59(75.6)$ & \\
\hline
\end{tabular}

IRG1, immune responsive gene 1.

with IRG1 low expression ( $\mathrm{n}=69$ ) had a long RFS compared with patients with high IRG1 expression $(\mathrm{n}=71, \mathrm{P}=0.0311$, Fig. $2 \mathrm{H})$. In the patients with WHO grade II and III, the subgroup with high IRG1 $(\mathrm{n}=42)$ expression had a lower RFS rate than the subgroup with low IRG1 expression $(n=48$, $\mathrm{P}=0.0164$, Fig. 2I).

IRG1-induced proliferation of glioma cells. To explore the effect of IRG1 on cancer cell growth, we used IRG1-specific siRNAs to inhibit IRG1 expression in the cancer cells. Both si-IRG1-1 and si-IRG1-2 downregulated IRG1 expression, but the inhibitory effect of si-IRG1-2 was more significant (Fig. 3A and B). Therefore, we used si-IRG1-2 in the further experiments. U251 and SHG-44 cells were transiently transfected with IRG1 and si-IRG1-2. The results of the MTT assays (Fig. 3C and D) revealed that the downregulation of IRG1 expression inhibited SHG- 44 cell proliferation by $43 \%(\mathrm{P}<0.01)$, whereas IRG1 overexpression promoted SHG-44 cell growth by $54 \%$ $(\mathrm{P}<0.01)$. The EDU assay showed that overexpression of IRG1 increased the percentage of proliferative cells from $\sim 23$ and 

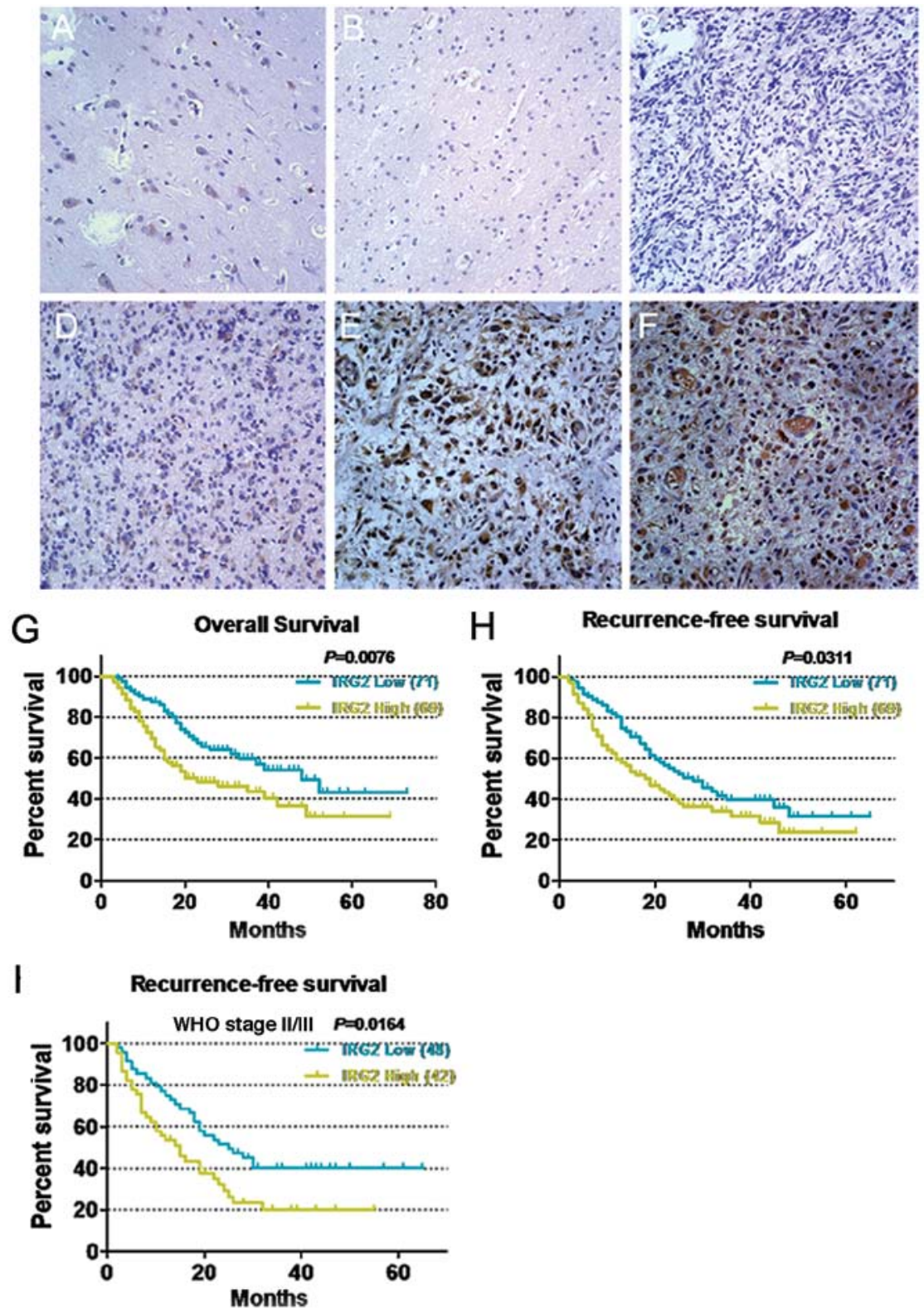

Figure 2. Cytoplasmic immune responsive gene 1 (IRG1) protein is a prognostic factor for post-surgical glioma patients. (A and B) Weak expression of IRG1 in normal brain tissues. (C) Weak expression of IRG1 in glioma tissues. (D) Medium expression of IRG1 in glioma tissues. (E and F) Strong staining of IRG1 in glioma tissues. (Original magnification x400). The relationship between IRG1 protein expression and (G) overall survival and (H) recurrence-free survival was investigated by Kaplan-Meier survival analysis in 140 glioma patients. (I) The relationship between IRGI protein expression and recurrence-free survival was investigated in patients subgrouped according to WHO grade II/III disease. The log-rank test was used to calculate P-values.

$\sim 25 \%$ to $\sim 64$ and $\sim 62 \%$ in the U251 and SHG-44 glioma cell lines; but inhibition of IRG1 using IRG1 siRNA decreased the percentage of proliferative cells in the glioma cells (from $\sim 23$ and $\sim 25 \%$ to $\sim 6$ and $\sim 9 \%$ in the U251 and SHG-44 cell lines) (Fig. 3E and F). Next, we examined the cell cycle distribution in the population of si-IRG1-2-transfected cells. Compared to the si-NC control, the U251 and SHG-44 glioma cell lines transfected with si-IRG1-2 displayed an increased proportion of cells entering the G1 phase and fewer cells in the $\mathrm{S}$ phase (Fig. 3G and $\mathrm{H} ; \mathrm{P}<0.01$ ). These results suggest that the growth-suppressive effect of si-IRG1 was partly due to G1 phase arrest. As shown by the colony formation assay, the si-IRG1-transfected U251 and SHG-44 cells formed fewer and smaller sized colonies than did the si-NC-transfected cells (Fig. 3I and J; $P<0.01$ ).
IRG1 knockdown reduces cell migration and invasion. Cell migration and invasion are integral steps in tumor development and metastasis. The wound healing assay showed that the lateral migration of cancer cells was inhibited by IRG1 knockdown (Fig. 4A and B). When we tested U251 and SHG-44 cell migration/invasion through an $8-\mu \mathrm{m}$-pore polycarbonate membrane with or without Matrigel pre-coating, we found that the knockdown of endogenous IRG1 significantly reduced the ability of U251 and SHG-44 cells to migrate and invade $(\mathrm{P}<0.05)$, compared to the si-NC cells (Fig. 4C-F).

IRG1 controls the expression of genes associated with cell cycle and epithelial-mesenchymal transition in glioma cells. To further investigate the mechanism of IRG1 regulation of cell proliferation, migration and invasion, we firstly examined the 

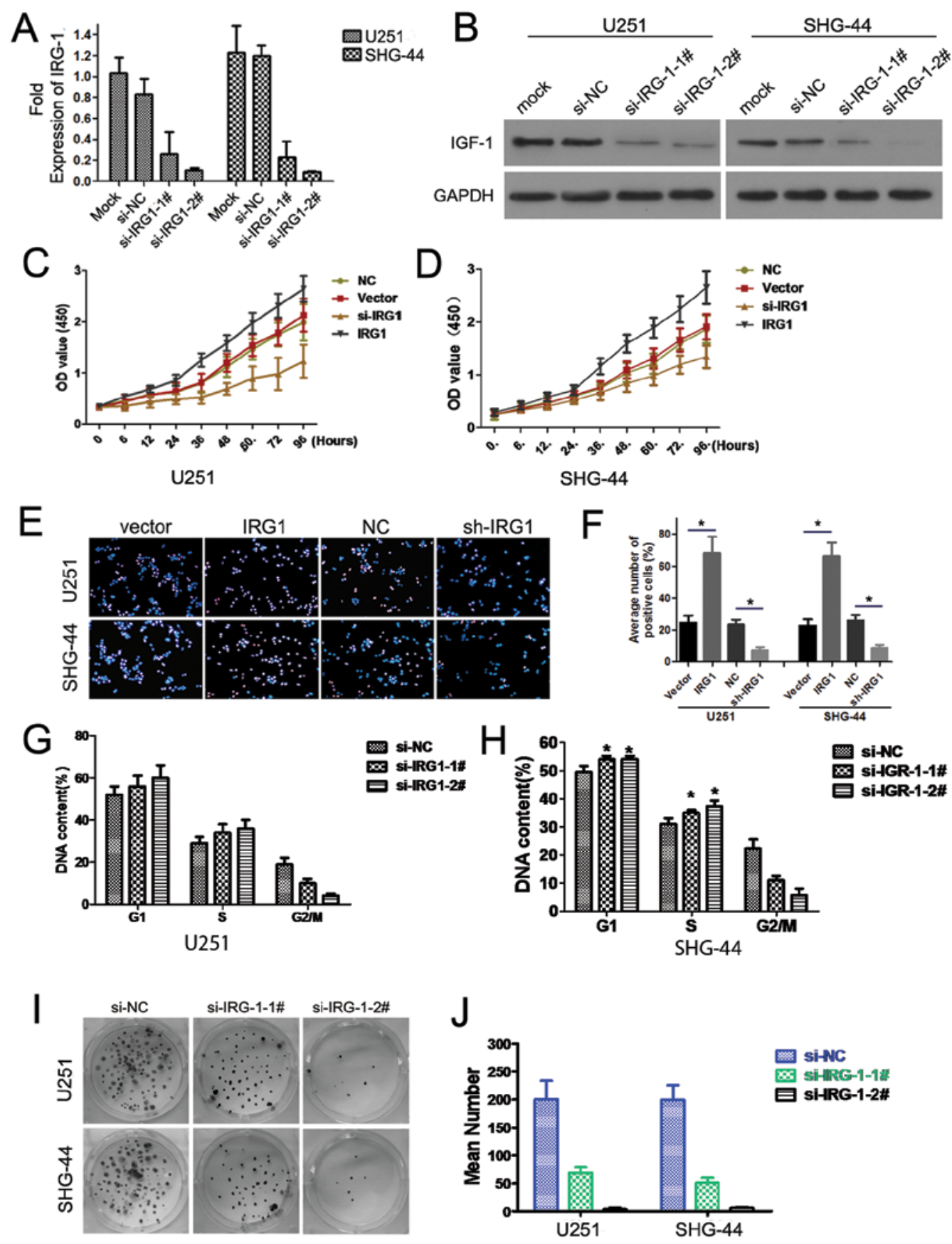

Figure 3. Immune responsive gene 1 (IRG1) induces the proliferation of glioma cells. U251 and SHG-44 cells were transfected with siRNA-NC, si-IRG1-1, and si-IRG1-2. (A) IRG1 mRNA expression was examined by qRT-PCR. Data are presented as means \pm SD of three independent experiments. (B) IRG1 protein expression was examined by western blotting. Growth of (C) U251 and (D) SHG-44 cells following knockdown or overexpression of IRG1 expression was examined by the MTT assay. (E and F) EDU assays showed that overexpression of IRG1 increased the percentage of proliferative cells in the U251 and SHG-44 glioma cell lines, while inhibition of IRG1 using IRG1 siRNA decreased the percentage of proliferative cells. (G and H) The cell cycle S/G1 transition was suppressed in cells with reduced IRG1 expression. Data are presented as means \pm SD of three independent experiments, "P<0.05. (I and J) Clone formation assay. Data are presented as means \pm SD of three independent experiments, ${ }^{*} \mathrm{P}<0.05$.

protein expression of the genes associated with cell cycle and epithelial-mesenchymal transition (EMT) in glioma U251 and SHG-44 cells with stably decreased IRG1 expression. Knockdown of endogenous IRG1 expression induced the inhibition of the tumor suppressor retinoblastoma protein $\mathrm{pRB}$ and the oncogenic cell cycle regulator transcription factor E2F1, while upregulating the expression of the tumor suppressor cyclindependent kinase inhibitor p21. However, the levels of the cyclin-dependent kinases CDK4 and CDK6 were depressed
(Fig. 5A). In addition, we found that IRG1 inhibition decreased the expression of the EMT marker genes snail, $\mathrm{N}$-cadherin, and vimentin $(13,14)$, while increasing the level of E-cadherin, an epithelial marker (Fig. 5B). IRG1 suppression did not promote the transition from epithelial to mesenchymal morphology in glioma SHG-44 cells.

Several studies have provided evidence that secretion of cytokines and growth factors, many of them encoded by the NF- $\mathrm{kB}$ target genes, is critical for constitutive activation 

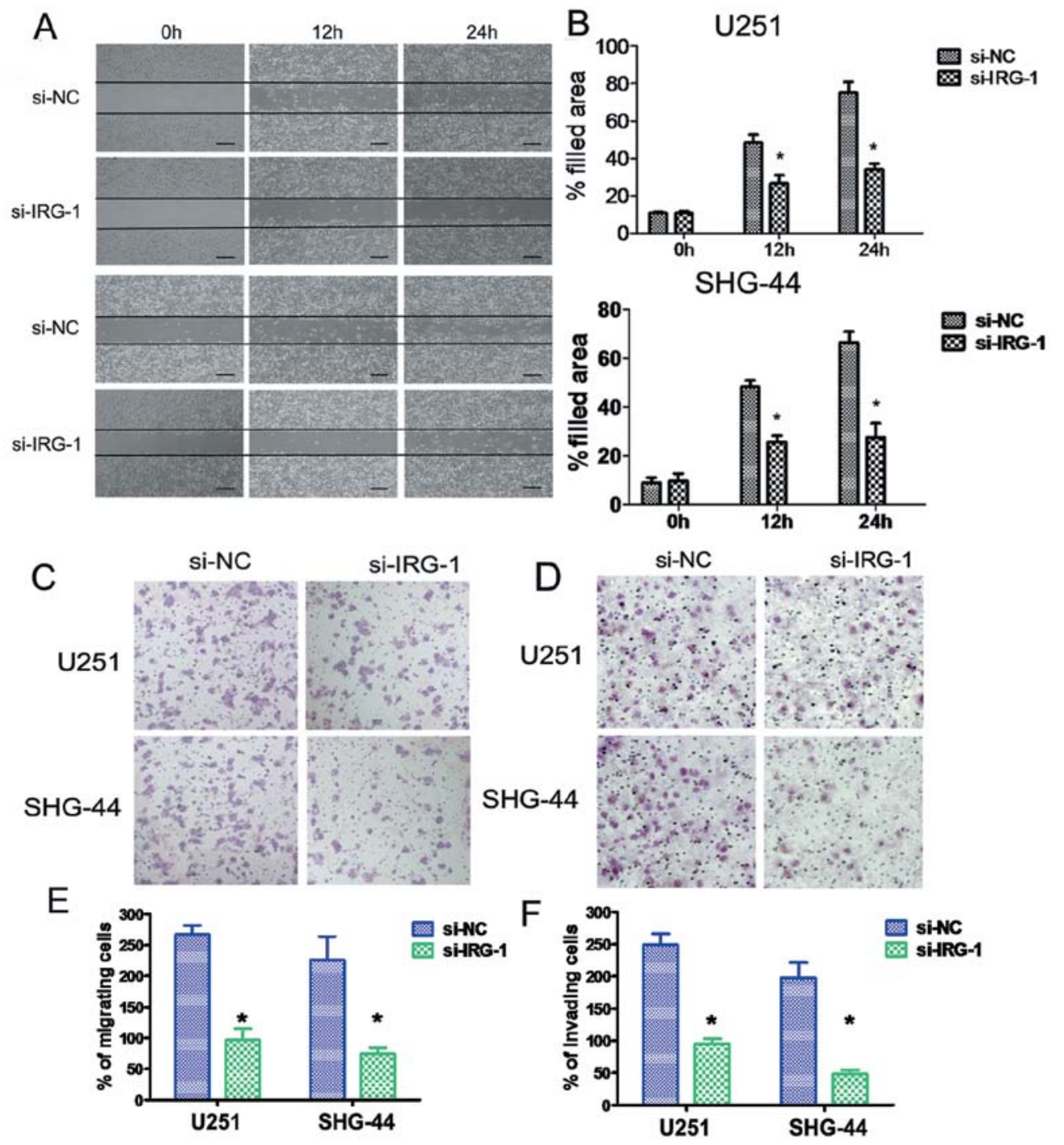

Figure 4. Immune responsive gene 1 (IRG1) knockdown reduces cell migration and invasion. (A and B) Wound healing assay. Confluent cell monolayers were scratched using a sterile pipette tip. At 12 and $24 \mathrm{~h}$ after wound infliction, cell migration into the scraped area was photographed. Wound healing was quantified by measuring the area occupied by the migrated cells and was expressed as a percentage. Data are presented as means \pm SD of three independent experiments, ${ }^{*} \mathrm{P}<0.05$. (C) Cell migration assays were performed using 12-well Transwell plates. Representative images show the density of the migrated cells. (E) Bar graph shows the quantification of cell migration. Data are presented as means \pm SD of three independent experiments, ${ }^{*} \mathrm{P}<0.05$. (D) Cell invasion assays were performed using 12-well Transwell plates. Representative images show the density of the invaded cells. (F) Bar graph shows the quantification of the cell invasion. Data are presented as means $\pm \mathrm{SD}$ of three independent experiments, ${ }^{*} \mathrm{P}<0.05$

of NF- $\mathrm{NB}$ in cancer cells (15). There has been an increasing interest in the role of $\mathrm{NF}-\kappa \mathrm{B}$ in the maintenance of established cancers, where it is frequently constitutively induced and plays a major role in the transcriptional activation of oncogenic genes (16). NF- $\kappa \mathrm{B}$ signaling involves degradation of the $\mathrm{NF}-\kappa \mathrm{B}$ inhibitor proteins $\mathrm{I} \kappa \mathrm{Bs}$ through phosphorylation by the I $\mathrm{B}$ kinase (IKK). In the past study, we found that knockdown of IRG1 expression by using si-IRG1 decreased the levels of $\mathrm{NF}-\kappa \mathrm{B}$ and $\mathrm{IkB} \alpha$, as well as STAT3, which was shown to maintain constitutive NF- $\kappa \mathrm{B}$ activity in tumors $(10,16)$ (Fig. 5C). These results indicate that the abrogation of IRG1 expression in glioma cells inhibits $\mathrm{NF}-\kappa \mathrm{B}$-mediated signaling.

IRG1 contributes to in vivo xenograft tumor growth. In addition to examining the biological functions of IRG1 in vivo, we assessed the in vivo role of IRG1 by using a xenograft transplantation model. We subcutaneously injected sh-IRG1 and
IRG1 lentiviral infection U251 and SHG-44 cells or control cells into nude mice, and monitored tumor growth over a 40-day period. As shown in Fig. 6A and B, the average tumor volume in mice injected with the IRG1-depleted U251 cells was markedly (by $>60 \%$ ) reduced when compared to that in the control animals $(\mathrm{P}<0.05)$. Tumor weight analysis showed that sh-IRG1-transfected cells gave rise to significantly smaller tumors than did sh-vector-transfected cells $(\mathrm{P}<0.05)$ (Fig. 6C and D). These results confirmed the previously results in vitro, indicating that expression of IRG1 increases the cancer cell growth of gliomas.

\section{Discussion}

Glioma is a disease characterized by uncontrolled cell growth in brain tissues that may lead to metastases (17). It ultimately results in the death of the patient. Tumor development from 

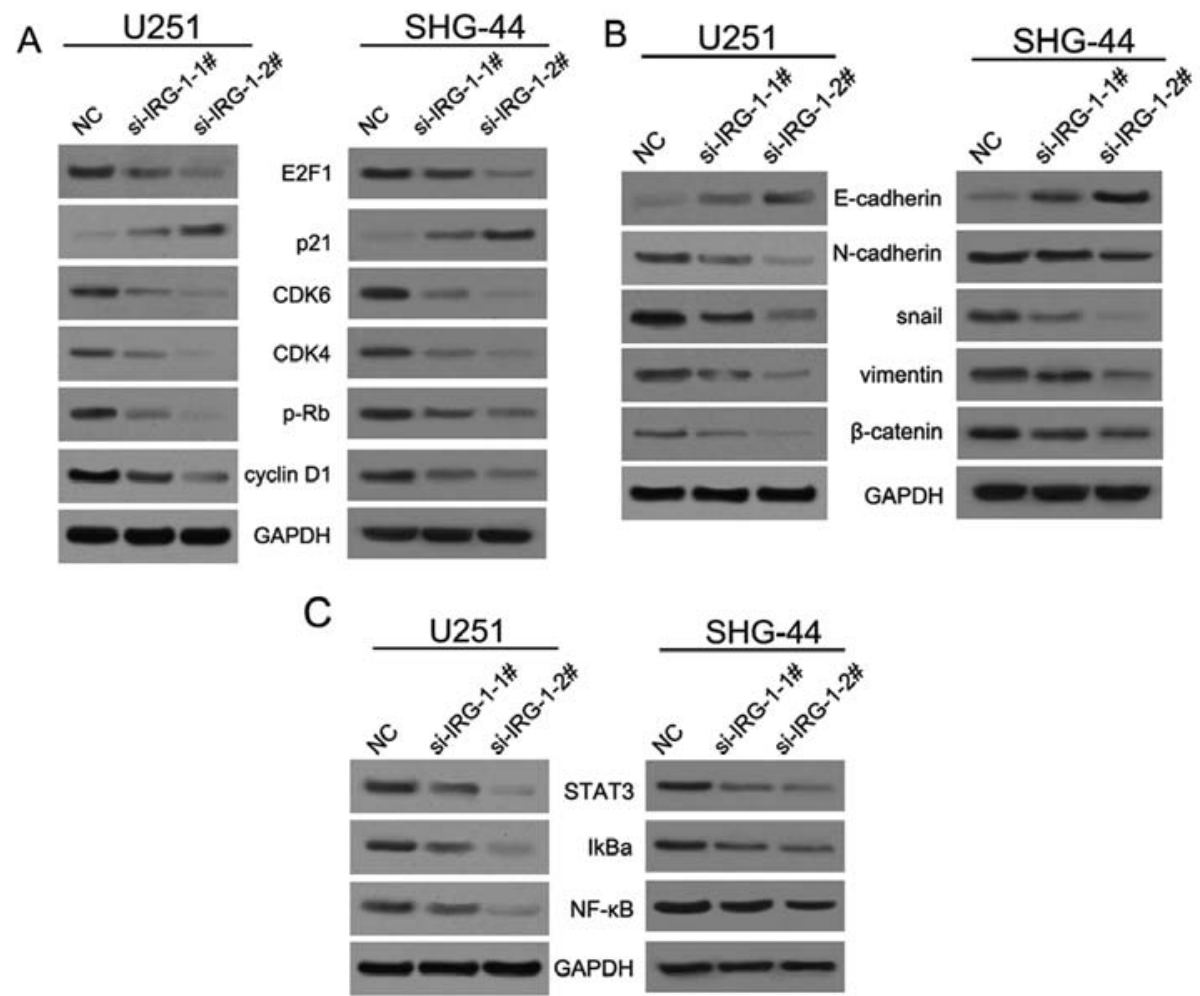

Figure 5. Immune responsive gene 1 (IRG1) controls the expression of cell cycle- and EMT-associated genes in glioma cells. Protein expression in U251 and SHG-44 glioma cells with reduced IRG1 levels was analyzed by western blotting. GAPDH was used as a loading control. (A) Expression of cell cycle regulatory proteins. (B) Expression of epithelial and mesenchymal markers. (C) Expression of STAT3, IкB $\alpha$ and NF-кB.

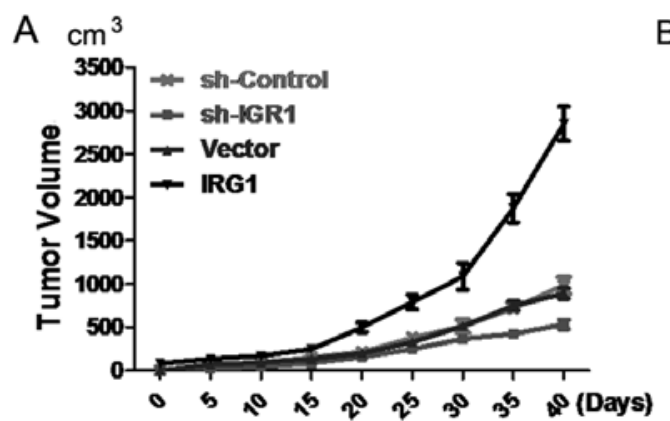

C

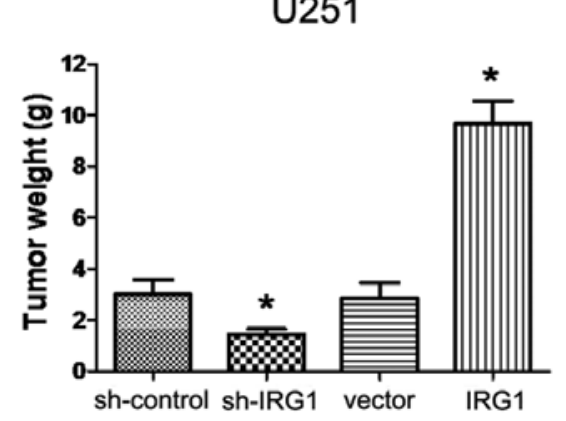

B

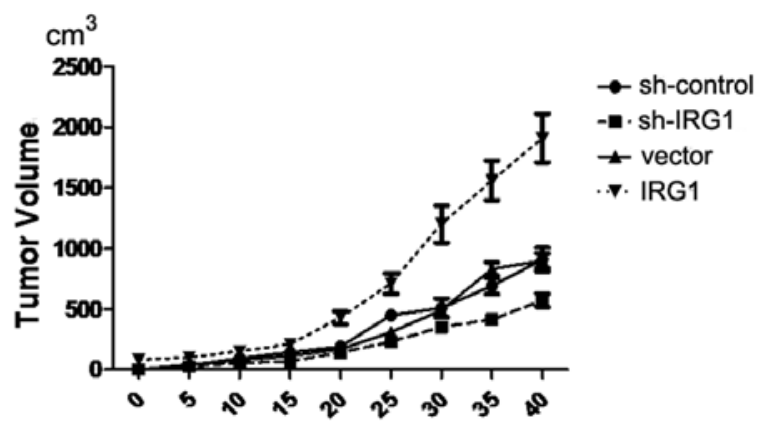

D

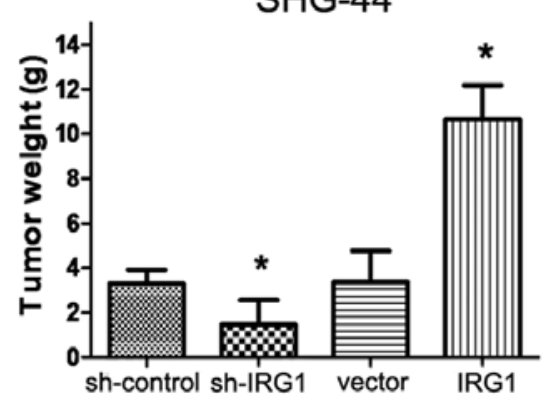

Figure 6. IRG1 contributes to in vivo xenograft tumor growth. (A and B) Tumor volumes were measured every four days. Each point represents the mean \pm SD of tumor volumes. (C and D) After five weeks, the mice were sacrificed and the tumors were removed and weighed. Results are shown as means \pm SD of tumor weights.

the stages of formation and growth to those of invasion and metastasis is a complex process that often involves changes in the tissue microenvironment, gene mutations, angiogenesis, and other pathological processes. In this series of events, pro-inflammatory proteins, including various cytokines and transcription factors, play a very important role (18). 
Inflammatory regulators are associated not only with early stages of tumor growth but also with malignant cell migration, invasion and metastasis (19).

The IRGl gene plays a crucial role in the orchestration of immune responses to infection (20). To clarify the role of IRG1 in glioma pathogenesis, we analyzed IRG1 protein expression in glioma and normal tissues by immunohistochemistry. We found that the IRG1 expression levels were higher in glioma tumors compared to those in normal brain tissues. Increased IRG1 expression was associated with disease progression and poor prognosis in glioma patients. Furthermore, inhibition of IRG1 expression upregulated the tumor suppressor p21 gene, and this may have caused inhibition of cell proliferation and migration. These results suggest that the molecular mechanisms underlying IRG1 activity may be associated with pro-inflammatory factors and EMT-related proteins.

The transcription factor E2F1 was initially identified as an adenoviral E2 promoter-binding cellular factor-1 induced by adenoviral E1A oncoprotein (21). E2F1 ectopic expression is sufficient to promote the G1/S phase transition in the cell cycle. Our findings indicate that IRG1 knockout can suppress E2F1 expression. At present, cumulative evidence points to the members of the cyclin D/CDK4/CDK6/pRB cell cycle regulatory pathway as potential targets for cancer therapy (22). Both CDK4 and CDK6 encode cyclin-dependent serine-threonine kinases that respond to mitogenic or pro-proliferative stimuli by complexing with D-type cyclins and phosphorylating the tumor suppressor protein $\mathrm{pRB}$. The phosphorylated $\mathrm{pRB}$ is released from the complex with E2F transcription factors, enabling E2F to initiate the transcription of genes required for G1/S cell cycle progression, and ultimately cellular proliferation (23). Here, we found that knockdown of endogenous IRG1 expression reduced the activation of CDK4, CDK6, and other oncogenic cell cycle regulators, including cyclin D1 and pRd, and increased the expression of tumor suppressors, including p21. Our results indicate that IRG1 may influence cell cycle transition by mediating the transcription of cell cycle regulatory proteins.

Previous studies have shown that snail, N-cadherin, and vimentin are markers of EMT (22). In this study, we found that the downregulation of IRG1 significantly inhibited the expression of snail, N-cadherin and vimentin in glioma SHG-44 cells. The in vitro Transwell cell migration assay and in vivo xenograft model showed that the downregulation of IRG1 expression significantly repressed the migration and pulmonary metastasis of glioma cells. Our studies showed that IRG1 inhibition promoted the expression of the epithelial marker E-cadherin, while reducing the levels of the mesenchymal markers snail, $\mathrm{N}$-cadherin, and vimentin in glioma cells. These data suggest that IRG1 may increase EMT and cancer metastasis. Several studies have provided evidence that secretion of pro-inflammatory cytokines and growth factors is critical for constitutive activation of NF- $\kappa \mathrm{B}$ in cancer cells (24-26). The reduced expression of IRG1 in glioma cells may have caused inhibition of $\mathrm{NF}-\kappa \mathrm{B}$ signaling, suggesting that IRG1 can be involved in carcinogenesis through NF- $\mathrm{KB}$ signaling as well.

In summary, our study revealed that the cytoplasmic expression of IRG1 was significantly upregulated in glioma and that it is an important prognostic factor for glioma patients. Our in vitro and in vivo data indicate that IRG1 is involved in proliferation, invasion, and migration of cancer cells through multiple molecular mechanisms, including regulation of $\mathrm{NF}-\kappa \mathrm{B}$ signaling, the EMT pathway, and cell cycle transition, suggesting that IRG1 plays an important role in the development of gliomas.

\section{Acknowledgements}

The authors thank Dr Jun Wu for his support and assistance with the preparation of the manuscript. This study was supported by grants from National Natural Science Foundation of China (no. 81172416).

\section{References}

1. Hamza MA and Gilbert M: Targeted therapy in gliomas. Curr Oncol Rep 16: 379, 2014.

2. Kaufmann JK and Chiocca EA: Glioma virus therapies between bench and bedside. Neuro Oncol 16: 334-351, 2014.

3. Lohkamp B, Bauerle B, Rieger PG and Schneider G: Threedimensional structure of iminodisuccinate epimerase defines the fold of the MmgE/PrpD protein family. J Mol Biol 362: 555-566, 2006.

4. Lee CG, Demarquoy J, Jackson MJ and O'Brien WE: Molecular cloning and characterization of a murine LPS-inducible cDNA. J Immunol 152: 5758-5767, 1994.

5. Gautam A, Dixit S, Philipp MT, et al: Interleukin-10 alters effector functions of multiple genes induced by Borrelia burgdorferi in macrophages to regulate Lyme disease inflammation. Infect Immun 79: 4876-4892, 2011.

6. Li Y, Zhang P, Wang C, et al: Immune responsive gene 1 (IRG1) promotes endotoxin tolerance by increasing A20 expression in macrophages through reactive oxygen species. J Biol Chem 288: 16225-16234, 2013.

7. Lee CG, Jenkins NA, Gilbert DJ, Copeland NG and O'Brien WE: Cloning and analysis of gene regulation of a novel LPS-inducible cDNA. Immunogenetics 41: 263-270, 1995.

8. Shi S, Blumenthal A, Hickey CM, Gandotra S, Levy D and Ehrt S: Expression of many immunologically important genes in Mycobacterium tuberculosis-infected macrophages is independent of both TLR 2 and TLR4 but dependent on IFN-alphabeta receptor and STAT1. J Immunol 175: 3318-3328, 2005.

9. Muccioli M, Sprague L, Nandigam H, Pate M and Benencia F: Toll-like receptors as novel therapeutic targets for ovarian cancer. ISRN Oncol 2012: 642141, 2012.

10. Hall CJ, Boyle RH, Astin JW, et al: Immunoresponsive gene 1 augments bactericidal activity of macrophage-lineage cells by regulating $\beta$-oxidation-dependent mitochondrial ROS production. Cell Metab 18: 265-278, 2013.

11. Chiba Y, Mizoguchi I, Mitobe K, et al: IL-27 enhances the expression of TRAIL and TLR3 inhuman melanomas and inhibits their tumor growth in cooperation with a TLR3 agonist poly(I:C) partly in a TRAIL-dependent manner. PLoS One 8: e76159, 2013

12. Song Q, Li Y, Zheng X, et al: MTA1 contributes to actin cytoskeleton reorganization and metastasis of nasopharyngeal carcinoma by modulating Rho GTPases and Hedgehog signaling. Int J Biochem Cell Biol 45: 1439-1446, 2013.

13. Lathia JD, Li M, Hall PE, et al: Laminin alpha 2 enables glioblastoma stem cell growth. Ann Neurol 72: 766-778, 2012.

14. Cheng L, Huang Z, Zhou W, et al: Glioblastoma stem cells generate vascular pericytes to support vessel function and tumor growth. Cell 153: 139-152, 2013.

15. Islam KN, Bae JW, Gao E and Koch WJ: Regulation of nuclear factor $\kappa \mathrm{B}(\mathrm{NF}-\kappa \mathrm{B})$ in the nucleus of cardiomyocytes by $\mathrm{G}$ protein-coupled receptor kinase 5 (GRK5). J Biol Chem 288: 35683-35689, 2013.

16. Cheah SC, Lai SL, Lee ST, Hadi AH and Mustafa MR: Panduratin A, a possible inhibitor in metastasized A549 cells through inhibition of NF-kappa B translocation and chemoinvasion. Molecules 18: 8764-8778, 2013.

17. Zhao L, Wang H, Sun X and Ding Y: Comparative proteomic analysis identifies proteins associated with the development and progression of colorectal carcinoma. FEBS J 277: 4195-4204, 2010 . 
18. Wang F, Qiao Y, Yu J, et al: FBX8 acts as an invasion and metastasis suppressor and correlates with poor survival in hepatocellular carcinoma. PLoS One 8: e65495, 2013.

19. Huang J, Ye X, Guan J, et al: Tiam1 is associated with hepatocellular carcinoma metastasis. Int J Cancer 132: 90-100, 2013.

20. Michelucci A, Cordes T, Ghelfi J, et al: Immune-responsive gene 1 protein links metabolism to immunity by catalyzing itaconic acid production. Proc Natl Acad Sci USA 110: 7820 $7825,2013$.

21. Kovesdi I, Reichel R and Nevins JR: E1A transcription induction: enhanced binding of a factor to upstream promoter sequences. Science 231: 719-722, 1986.

22. Wang WJ, Wu SP, Liu JB, et al: MYC regulation of CHK1 and CHK2 promotes radioresistance in a stem cell-like population of nasopharyngeal carcinoma cells. Cancer Res 73: 1219-1231, 2013.
23. Lee HH, Lee SR and Leem SH: Tristetraprolin regulates prostate cancer cell growth through suppression of E2F1. J Microbiol Biotechnol 24: 287-294, 2013.

24. Kim MK, Kang YJ, Kim DH, et al: A novel hydroxamic acid derivative, MHY218, induces apoptosis and cell cycle arrest through downregulation of NF- $\kappa \mathrm{B}$ in HCT116 human colon cancer cells. Int J Oncol 44: 256-264, 2014.

25. Kim A, Yim NH, Im M, Jung YP, Kim T and Ma JY: Suppression of the invasive potential of highly malignant tumor cells by $\mathrm{KIOM}-\mathrm{C}$, a novel herbal medicine, via inhibition of NF- $\mathrm{\kappa B}$ activation and MMP-9 expression. Oncol Rep 31: 287-297, 2014.

26. Zhang W and Grivennikov SI: Top Notch cancer stem cells by paracrine $\mathrm{NF}-\kappa \mathrm{B}$ signaling in breast cancer. Breast Cancer Res 15: 316, 2013. 